Review

\title{
Aspiration Pneumonia and the Traditional Chinese Medicine Banxia Houpu Tang
}

Koh Iwasaki *

Natori Kumanodoh Hospital, Natori, Japan; E-Mail: iwasaki.koh67@gmail.com

* Correspondence: Koh Iwasaki; E-Mail: iwasaki.koh67@gmail.com

Academic Editor: David G Smithard

Special Issue: Stroke in Older Adults

\section{OBM Geriatrics}

2020, volume 4, issue 4

doi:10.21926/obm.geriatr.2004136
Received: August 25, 2020

Accepted: October 21, 2020

Published: October 27, 2020

\begin{abstract}
Aspiration pneumonia is one of the most common reasons for death in stroke patients. Recently, a traditional herbal medicine banxia houpu tang is remarked to prevent the aspiration pneumonia. This review tells the history of study of banxia houpu tang for prevent aspiration pneumonia.

\section{Keywords}

Aspirartion pneumonia; banxia houpu tang; traditional Chinese medicine; the swallowing reflex; the cough reflex
\end{abstract}

Aspiration pneumonia is one of the most common reasons for death in stroke patients. In the patients with aspiration pneumonia, marked depressions in the swallowing and cough reflexes are observed [1]. Once this condition occurs, antibiotics and inanition are the only treatment options. Even after improvement, the patients are often unable to consume food orally and require tube feeding. Although tube feeding is good for nutritional management, it significantly reduces the quality of life (QOL) of the patient. Therefore, prevention of aspiration pneumonia as well as

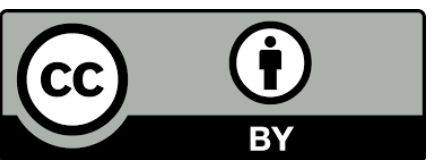

(C) 2020 by the author. This is an open access article distributed under the conditions of the Creative Commons by Attribution License, which permits unrestricted use, distribution, and reproduction in any medium or format, provided the original work is correctly cited. 
improvement of oral food intake is crucial for the QOL for these patients. Angiotensin-converting enzyme inhibitors (ACEI) are reported to prevent pneumonia in elderly patients [2]. The effects of ACEI on swallowing [3] and cough [4] reflexes may prevent pneumonia. However, whether receiving ACEI is capable of reducing post-stroke aspiration pneumonia in the current clinical environment remains unclear [5].

Banxia Houpu Tang (半夏厚朴湯, BHT) is an ancient traditional Chinese medicine (TCM) with a medical usage history of over 2000 years. In Japan, BHT extracts $(4.5 \mathrm{~g})$ are prepared from a mixture of five dried plants: Pinelliae tuber (Pinellia ternate Breitenbach), $6 \mathrm{~g}$; Hoelen (Poria cocos Wolf), 3 g; Magnoliae cortex (Magnolia obovata Thunberg), $3 \mathrm{~g}$; Perillae herba (Perilla frutescens Britton ar. Acuta Lubo), $2 \mathrm{~g}$; and Zingiberis rhizoma (Zingiber officcinale Roscoc), $1 \mathrm{~g}$. This medical-grade extract powder is commercially available as the traditional medicine named Hange-Koboku Toh (the Japanese name for BHT) from Tsumura Co. Ltd. (Tokyo, Japan). Each of the five herbs was first registered in the Pharmacopoeia of Japan, and the herb quality was controlled as specified in the Pharmacopoeia of Japan. Generally, this medicine is used for neurotic disorders and depression. In an ancient TCM textbook, Jinkuiyaolue (金貴要略) states that this medicine was effective in women who complained of a feeling similar to a fruit seed being stuck in the throat, which is nowadays considered a symptom of throat dysesthesia.

In our randomized control study [6], BHT was observed to ameliorate the swallowing [7] and cough [8] reflexes and reduce the risk of aspiration pneumonia in stroke patients (Figure 1 and 2). In a recent double-blinded randomized control study [9], BHT was observed to reduce aspiration pneumonia. Therefore, BHT is a useful tool for preventing aspiration pneumonia in stroke patients. Unfortunately, according to the Cochrane Database, there is no evidence that the other TCM herbs and acupuncture improved aspiration pneumonia [10]. However, a meta-analysis has reported that acupuncture is effective in dysphagia in post-stroke patients [11].

Figure 1 Study protocol. 


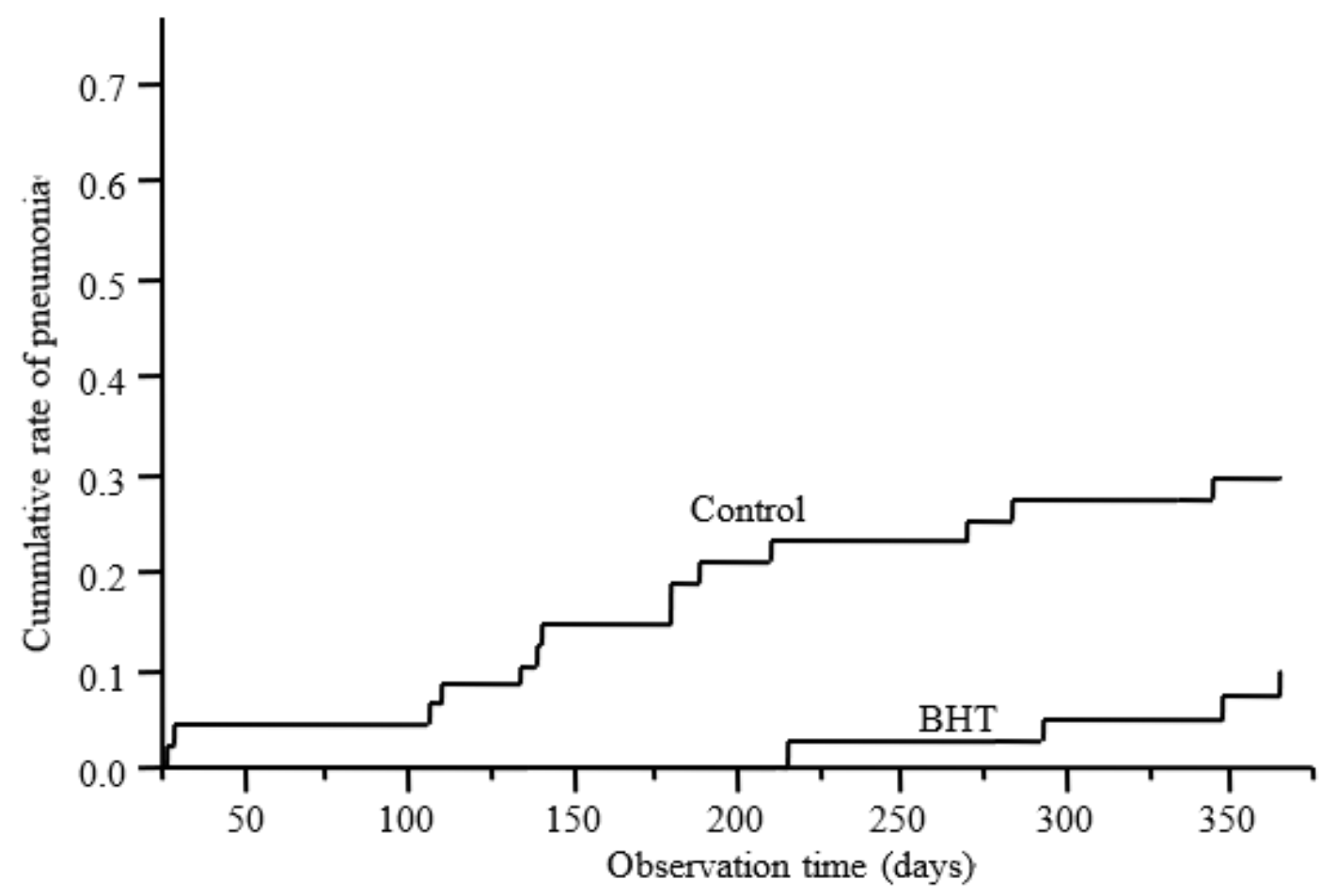

Figure 2 Accumulated rate of pneumonia onset. Pneumonia developed in four $(9.1 \%)$ patients in the BHT group and 14 (29.2\%) patients in the control group. There was a statistically significant difference in the rate of pneumonia $(p=0.008)$ between the two groups. Taken from an earlier study [8].

According to the global standard perspective, tube feedings should be performed only in certain stroke patients, particularly the brainstem stroke patients. However, in Japan, the doctors in acute phase hospitals often prescribe tube feeding to stroke and dementia patients as the acute phase hospitals lack time to wait for the dysphagia improvement to occur. Nonetheless, this is a corrupt practice in Japan, as a consequence of which, several stroke patients who are unable to consume food orally are managed on tube feeding for a long time. Although tube feeding has an advantage of nutritional management, it significantly reduces the quality of life of the patients. Therefore, discontinuing tube feeding and switching to oral intake is crucial for improving the quality of life of the patients and maintaining their dignity. In this context, the present study involved shifting to nutritional management by oral intake after receiving tube feeding through a nasogastric tube or gastrostomy for over 12 months [12]. Fourteen participants (male/female ratio = 6: 8; age = $83.9 \pm 2.6$ years) attempted to discontinue tube feeding and resume oral feeding. The diagnosis, duration of tube feeding, gender, swallowing reflex time, and the consciousness levels (Kohnan score) [13] of these participants were analyzed. The patients who required over $4 \mathrm{~s}$ for the swallowing reflex were administered BHT. Seven of the 14 patients recovered oral feeding, while seven failed in the attempt. The mean age, duration of tube feeding, swallowing reflex time, and gender demonstrated no significant differences. Consciousness level exhibited a significant difference between the success group and the failure group. BHT significantly improved the swallowing reflex time in seven patients. The results revealed that half of the patients who received tube feeding for over 12 months were able to return to oral intake, and there was a difference in the state of consciousness represented by the Konan score between the successful cases and the 
failed cases. Therefore, in patients with a good level of consciousness, it may be possible to discontinue tube feeding and resume oral feeding.

\section{Conclusion}

As mentioned above, BHT is one choice to improve dysphagia in the stroke patients. Some methods to improve swallowing reflex for example ACE inhibitors and cilostazol. We did not know yet how to use these methods in complex. In daily practice, we combined ACE inhibitors and BHT when the patient has hypertension, and cilostazol and BHT to patient having cardiac coronary disease. But we do not know interaction with each other. Farther examinations are needed.

\section{Author Contributions}

Koh Iwasaki completed all work for this manuscript.

\section{Competing Interests}

The author has declared that no competing interests exist.

\section{References}

1. Ely EW, Haponik EF. Pneumonia in the elderly. J Thorac Imaging. 1991; 6: 45-61.

2. Arai T, Sekizawa K, Ohrui T, Fujiwara $H$, Yoshimi $N$, Matsuoka $H$, et al. ACE inhibitors and protection against pneumonia in elderly patients with stroke. Neurology. 2005; 64: 573-574.

3. Nakayama K, Sekizawa K, Sasaki H. ACE inhibitors and swallowing reflex. Chest. 1998; 113: 1425.

4. Israli Z, Hall W. Cough and angioneurotic edema associated with angiotensin-converting enzyme inhibitor therapy: A review of the literature and pathophysiology. Ann Intern Med. 1992; 117: 234-242.

5. Kumazawa R, Jo T, Matsui H, Fushimi K, Yasunaga H. Association between angiotensinconverting enzyme inhibitors and post-stroke aspiration pneumonia. J Stroke Cerebrovasc Dis. 2019; 28: 104444.

6. Iwasaki K, Kato S, Monma Y, Niu K, Ohrui T, Okitsu R, et al. A pilot study of banxia houpu tang, a traditional Chinese medicine, for reducing pneumonia risk in older adults with dementia. J Am Geriatr Soc. 2007; 55: 2035-2040.

7. Iwasaki K, Wang $Q$, Nakagawa $T$, Suzuki T, Sasaki $H$. The traditional Chinese medicine banxia houpo tang improves swallowing reflex. Phytomedicine. 1999; 6: 103-106.

8. Iwasaki K, Cyong JC, Kitada S, Kitamura H, Ozeki J, Satoh Y, et al. A traditional Chinese herbal medicine, banxia houpo tang, improves cough reflex of patients with aspiration pneumonia. J Am Geriatr Soc. 2002; 50: 1751-1752.

9. Iwasaki K, Kato S, Monma Y, Niu K, Ohrui T, Okitsu R, et al. A pilot study of banxia houpu tang, a traditional Chinese medicine, for reducing pneumonia risk in older adults with dementia. J Am Geriatr Soc. 2007; 55: 2035-2040.

10. Kawago K, Nishibe T, Shindo S, Inoue H, Motohashi S, Akasaka J, et al. A double-blind randomized controlled trial to determine the preventive effect of hangekobokuto on aspiration pneumonia in patients undergoing cardiovascular surgery. Ann Thorac Cardiovasc Surg. 2019; 25: 318-325. 
11. Xie Y, Wang L, He J, Wu T. Acupuncture for dysphagia in acute stroke. Cochrane Database Syst Rev. 2008; 3: CD006076.

12. Li LX, Deng K, Qu Y. Acupuncture treatment for post-stroke dysphagia: An update meta-analysis of randomized controlled trials. Chin J Integr Med. 2018; 24: 686-695.

13. Nogami T, Koh I. Recovery of oral feeding in Japanese elderly people after long-term tube feeding: A challenge. J Fam Med Prim Care. 2020; 9: 3977-3980.

14. Sato $M$, Sugimoto $M$, Yamaguchi $K$, Kawaguchi T. Evaluation of nursing interventions using minimally invasive assessments methods for patients in a persistent vegetative state. Psychogeriatrics. 2017; 17: 406-413.

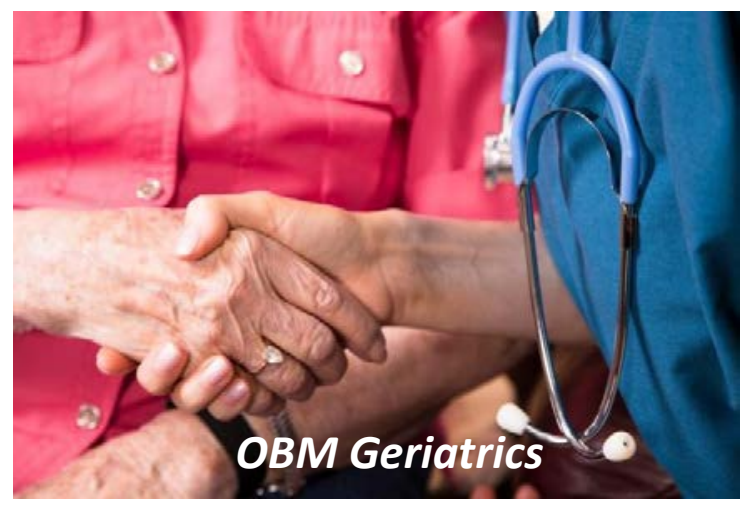

Enjoy $O B M$ Geriatrics by:

1. Submitting a manuscript

2. Joining in volunteer reviewer bank

3. Joining Editorial Board

4. Guest editing a special issue

For more details, please visit:

http://www.lidsen.com/journals/geriatrics 